\title{
ВЛИЯНИЕ ВЕЛИЧИНЫ ИНДУКТИВНОСТИ ЛИНЕИНОГО ДРОССЕЛЯ НА СВОЙСВА ТИРИСТОРНОГО РЕГУЛЯТОРА ПЕРЕМЕННОГО НАПРЯЖЕНИЯ С ПОДАВЛЕННЫМИ ЧЕТНЫМИ ГАРМОНИКАМИ
}

В целях улучшения спектрального состава тока тиристорных регуляторов переменного напряжения применяются схемы с линейной индуктивностью в контуре встречно-параллельно подключенных тиристоров $\left[{ }^{1-3}\right]$. Особенностью подобных схем является свободное прохождение через линейный дроссель нечетных гармоник тока. Такая схема проанализирована лишь в идеализированном случае, когда индуктивность линейного дросселя бесконечно велика. В настоящей статье учитывается конечное значение этой индуктивности.

Схема тиристорного регулятора переменного напряжения изображена на рис. 1. Переменное синусоидальное напряжение $u_{1}$ подается через встречно-параллельно подключенные тиристоры $T_{1}$ и $T_{2}$ на нагрузку $R$. Для заграждения тока четных гармоник последовательно с тиристорами подключены две магнитно-связанные индуктивности $L_{1}$ и $L_{2}$ в виде двухобмоточного линейного дросселя Др с центральным выводом. Благодаря согласному соединению индуктивностей $L_{1}$ и $L_{2}$ четные гармоники тока встречают индуктивное сопротивление дросселя, которое относительно нечетных гармоник тока отсутствует.

Четыре возможных режима работы подобного регулятора в зависимости от параметров схемы и угла отпирания тиристоров показаны на рис. $2, a, \sigma, \varepsilon$, г. Принятые здесь обозначения: $u_{1}-$ напряжение пи-

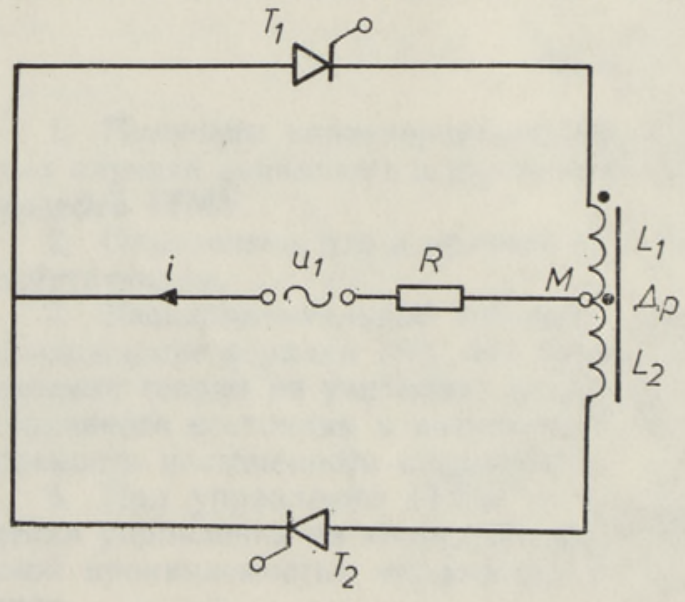

Рис. 1. тания, $i$ - ток нагрузки, $t_{s}-$ момент отпирания одного тиристора, $t_{a}$ - момент запирания другого тиристора, $i_{s}-$ ток нагрузки, соответствующий $t_{s}$ (после скачка тока), $i_{a}$ - ток нагрузки, соответствующий $t_{a}$.

При анализе пренебрегаем активными сопротивлениями обмоток дросселя Др и прямыми перепадами напряжения на отпертых тиристорах. Индуктивности $L_{1}=L_{2}=L=M$. Нагрузка $R$ - чисто активная. Тиристорами управляют симметрично. 
При работе схемы можно выделить два этапа.

I э та п. Оба тиристора отперты. Следовательно, перепады напряжения на тиристорах и на дросселе отсутствуют (короткозамкнутый контур). Выходное напряжение полностью соответствует входному. I этап имеет место при $\omega t_{s} \leqslant \omega t \leqslant \omega t_{a}$ и характеризуется уравнениями:

$$
\begin{aligned}
& i R=U_{1 m} \sin \omega t, \\
& i_{s}=\frac{U_{1 m} \sin \omega t_{s}}{R}, \\
& i_{a}=\frac{U_{1 m} \sin \omega t_{a}}{R} .
\end{aligned}
$$

II э т а п. Один тиристор отперт, другой заперт:

$$
L \frac{d i}{d t}+R i=U_{1 m} \sin \omega t .
$$

Поскольку

$$
i=\mathrm{e}^{-\int \frac{R}{L} d t}\left[\int \frac{U_{1 m}}{L} \sin \omega t \cdot \mathrm{e}^{\int \frac{R}{L} d t} d t+C_{1}\right],
$$

To

$$
i=F\left(\frac{R}{L} \sin \omega t-\omega \cos \omega t\right)+C_{1} \mathrm{e}^{-\frac{R}{L} t},
$$

где

$$
F=\frac{U_{1 m}}{L\left[\left(\frac{R}{L}\right)^{2}+\omega^{2}\right]} .
$$

Уравнения (3) и (5) содержат три неизвестных: $i_{a}, \omega t_{a}, C_{1}$. Примем, что

$$
i_{a}=i_{\omega t_{a}}=i_{\pi+\omega t_{s}}
$$

Последнее условие гарантируется отсутствием активного сопротивления в короткозамкнутом контуре, который сохраняет неизменный ток.

Получаем три уравнения с тремя неизвестными:

$$
\begin{aligned}
i_{a} & =F\left(\frac{R}{L} \sin \omega t_{a}-\omega \cos \omega t_{a}\right)+C_{1} \mathrm{e}^{-\frac{R}{L} \frac{\omega t_{a}}{\omega}}, \\
i_{a} & =F\left[\frac{R}{L} \sin \left(\pi+\omega t_{s}\right)-\omega \cos \left(\pi+\omega t_{s}\right)\right]+C_{1} \mathrm{e}^{-\frac{R}{L} \frac{\pi+\omega t_{s}}{\omega}}, \\
i_{a} & =\frac{U_{1 m} \sin \omega t_{a}}{R} .
\end{aligned}
$$

Введем обозначения

$$
\begin{aligned}
& A=F\left[\frac{R}{L} \sin \left(\pi+\omega t_{s}\right)-\omega \cos \left(\pi+\omega t_{s}\right)\right], \\
& B=\mathrm{e}^{-\frac{R}{L} \frac{\pi+\omega t_{s}}{\omega}}
\end{aligned}
$$

и решим равенство (9) относительно $C_{1}$, подставляя выражение (3): 


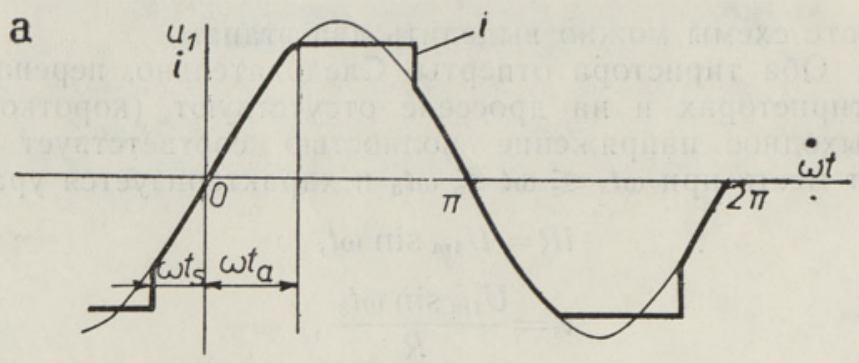

$\delta$

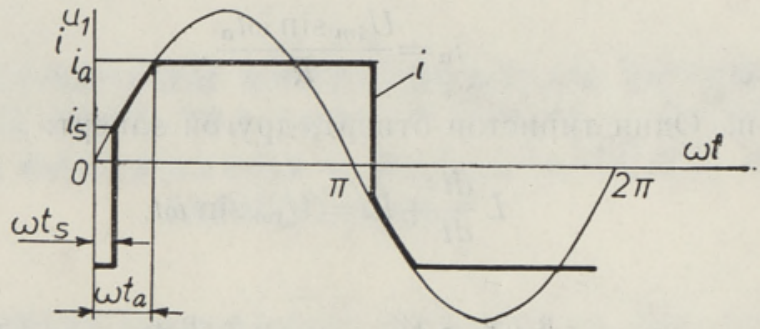

B

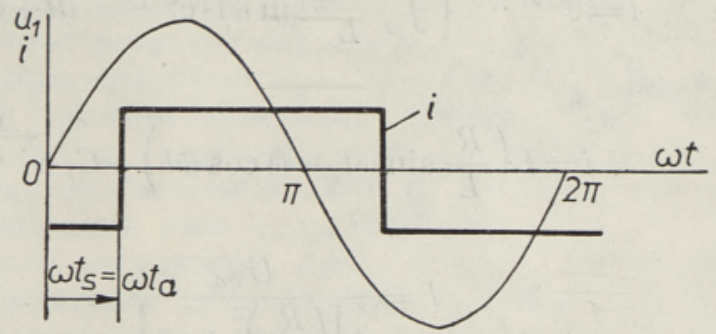

a

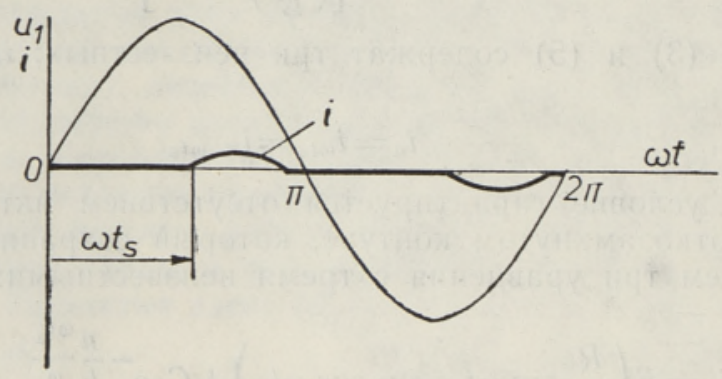

Рис. 2.

$$
C_{1}=\frac{U_{1 m} \sin \omega t_{a}}{R B}-\frac{A}{B} .
$$

Подстановка формулы (11) в соотношение (8) дает

$\frac{U_{1 m} \sin \omega t_{a}}{R}=F\left(\frac{R}{L} \sin \omega t_{a}-\omega \cos \omega t_{a}\right)+\left(\frac{U_{1 m} \sin \omega t_{a}}{R B}-\frac{A}{B}\right) \mathrm{e}^{-\frac{R}{L} \frac{\omega t_{a}}{\omega}}$

Поскольку последнее уравнение трансцендентное, то искомое $\omega t_{a}$ находится методом постепенного сближения на ЭВМ.

Проанализируем четыре возможных режима работы тиристорного регулятора. Первый и второй режимы характеризуются интервалом времени, когда оба тиристора отперты (рис. $2 a$, б) 


$$
\omega t_{a}>\omega t_{s}
$$

Кроме того, при первом режиме (рис. 2, a) угол отпирания тиристора $\omega t_{s}$ отрицателен. Для этих двух режимов справедливы приведенные выше формулы.

Третий режим наступает при увеличении глубины регулирования до определенного угла $\omega t_{s}=\alpha_{n}$, который зависит от постоянной времени регулятора:

$$
\tau=L / R
$$

Можно отметить, что при $\tau=\infty$

$$
\boldsymbol{\alpha}_{n}=\operatorname{arctg} 2 / \pi=32^{\circ} 29^{\prime} .
$$

Третий режим (рис. 2, в) характеризуется равенством

$$
\omega t_{s}=\omega t_{a} .
$$

Выходное напряжение стаповится в этот период приблизительно прямоугольным. Для нахождения предельного $\omega t_{a}=\omega t_{s}$ при переходе от второго режима к третьему приходится сравнивать найденное из уравнения (13) $\omega t_{a}$ с $\omega t_{s}$ : если $\omega t_{a} \leqslant \omega t_{s}$, то $\omega t_{a}=\omega t_{s}$.

Условие (7) принимает вид

$$
i_{a}=i_{\omega t_{s}}=i_{\pi+\omega t_{s}} .
$$

Уравнение (3) недействительно, и получаем два уравнения:

$$
\begin{gathered}
i_{a}=F\left(\frac{R}{L} \sin \omega t_{s}-\omega \cos \omega t_{s}\right)+C_{2} \mathrm{e}^{-\frac{R}{L} \frac{\omega t_{s}}{\omega}}, \\
i_{a}=A+C_{2} B=F\left[\frac{R}{L} \sin \left(\pi+\omega t_{s}\right)-\omega \cos \left(\pi+\omega t_{s}\right)\right]+C_{2} \mathrm{e}^{-\frac{R}{L} \frac{\pi+\omega t_{s}}{\omega}} .
\end{gathered}
$$

Отсюда

$$
C_{2}=\frac{F\left[-\frac{R}{L} \sin \omega t_{s}+\omega \cos \omega t_{s}+\frac{R}{L} \sin \left(\pi+\omega t_{s}\right)-\omega \cos \left(\pi+\omega t_{s}\right)\right]}{\mathrm{e}^{-\frac{R}{L} \frac{\omega t_{s}}{\omega}}-\mathrm{e}^{-\frac{R}{L} \frac{\pi+\omega t_{s}}{\omega}}} .
$$

Обозначаем

$$
\begin{aligned}
& D=\mathrm{e}^{-\frac{R}{L} \frac{\omega t_{s}}{\omega}}, \\
& E=\frac{R}{L}\left[\sin \left(\pi+\omega t_{s}\right)-\sin \omega t_{s}\right]+\omega\left[\cos \omega t_{s}-\cos \left(\pi+\omega t_{s}\right)\right], \\
& C_{2}=\frac{F E}{D-B}, \\
& i=F\left(\frac{R}{L} \sin \omega t-\omega \cos \omega t\right)+C_{2} \mathrm{e}^{-\frac{R}{L} t} .
\end{aligned}
$$

Четвертый режим (рис. 2, г) наступает при глубоком регулировании, когда в течение некоторого времени оба тиристора заперты и ток нагрузки равен нулю. Теперь дроссель подавляет и нечетные гармоники 
тока (последовательно с нагрузкой).

Здесь действительно уравнение (4), но в его решении появляется новая постоянная

$$
i=F\left(\frac{R}{L} \sin \omega t-\omega \cos \omega t\right)+C_{3} \mathrm{e}^{-\frac{R}{L} t} .
$$

Находим ее, учитывая, что в момент отпирания тиристора ток нагрузки равен нулю

$$
i_{s}=F\left(\frac{R}{L} \sin \omega t_{s}-\omega \cos \omega t_{s}\right)+C_{3} \mathrm{e}^{-\frac{R}{L} \frac{\omega t_{s}}{\omega}}=0
$$

откуда

$$
C_{3}=-\frac{F\left(\frac{R}{L} \sin \omega t_{s}-\omega \cos \omega t_{s}\right)}{\mathrm{e}^{-\frac{R}{L} \frac{\omega t_{s}}{\omega}}}
$$

Для нахождения $\omega t_{s}$ при переходе от третьего режима к четвертому необходимо найти момент изменения знака тока $i$ в уравнении (5a). Этот момент обозначим через $\omega t_{u}$. Задача решается на ЭВМ. Если $\omega t_{u} \leqslant \omega t_{s}+\pi$, то наступает четвертый режим.

Остается найти интегральные величины. 1. Эффективное значение тока нагрузки первых двух режимов:

$$
\begin{aligned}
I & =\sqrt{\frac{2}{T} \int_{0}^{T / 2} i^{2} d t}=\sqrt{\frac{1}{\pi} \int_{\omega t_{s}}^{\pi+\omega t_{s}} i^{2} d(\omega t)}= \\
& =\sqrt{\frac{1}{\pi}\left[\int_{\omega t_{s}}^{\omega t_{a}} i^{2} d(\omega t)+\int_{\omega t_{a}}^{\pi+\omega t_{s}} i^{2} d(\omega t)\right]},
\end{aligned}
$$

где $i$ в пределах $\omega t_{s} \rightarrow \omega t_{a}$ берется из уравнения (1), а в пределах $\omega t_{a} \rightarrow \pi+\omega t_{s}-$ из уравнения (5).

2. Аналогично находим среднее значение тока нагрузки для второго режима:

$$
\begin{aligned}
I_{\mathrm{cp}} & =\frac{2}{T} \int_{0}^{T / 2} i d t=\frac{1}{\pi} \int_{\omega t_{s}}^{\pi+\omega t_{s}} i d(\omega t)= \\
& =\frac{1}{\pi}\left[\int_{\omega t_{s}}^{\omega t_{a}} i d(\omega t)+\int_{\omega t_{a}}^{\pi+\omega t_{s}} i d(\omega t)\right] .
\end{aligned}
$$

Здесь следует обратить внимание на то, что при изменении $\omega t_{s}$ в обратном направлении (для достижения максимального тока нагрузки) $-\frac{\pi}{2} \leqslant \omega t_{s} \leqslant 0$ (первый режим) $I_{\text {ср }}$ сохраняет свое значение. Следовательно, в этой области уравнение (25) недействительно. Правильный результат получается при изменении пределов и знака первого слагаемого: 


$$
I_{\mathrm{cp}}=\frac{1}{\pi}\left[-\int_{\omega t_{s}}^{0} i d(\omega t)+\int_{0}^{\omega t a} i d(\omega t)+\int_{\omega t_{a}}^{\pi+\omega t_{s}} i d(\omega t)\right] .
$$

Решать последнее уравнение нет смысла, поскольку $I_{\text {ср }}$ постоянен.

3. Эффективное значение тока нагрузки для третьего режима:

$$
I=\sqrt{\frac{1}{\pi} \int_{\omega t_{s}}^{\pi+\omega t_{s}} i^{2} d(\omega t),}
$$

где $i$ берется из уравнения (5a).

4. Среднее значение тока нагрузки для третьего режима:

$$
I_{\mathrm{cp}}=\frac{1}{\pi} \int_{\omega t_{s}}^{\pi+\omega t_{s}} i d(\omega t) .
$$

При нахождении интегральных величин для четвертого режима необходимо определить момент запирания тиристоров $\omega t_{0}$ по (5б). Естественно, что уравнения для четвертого режима действительны только при $\omega t_{0} \leqslant \omega t_{s}+\pi$.

5. Эффективное значение тока нагрузки для четвертого режима

$$
I=\sqrt{\frac{1}{\pi} \int_{\omega t_{s}}^{\omega t_{0}} i^{2} d(\omega t)}
$$

где $i$ берется из уравнения (5б).

6. Среднее значение тока нагрузки для четвертого режима

$$
I_{\mathrm{cp}}=\frac{1}{\pi} \int_{\omega t_{s}}^{\omega t_{0}} i d(\omega t)
$$

7. Коэффициент формы

$$
K_{\Phi}=I / I_{\mathrm{cp}}
$$

8. Коэффициент мощности

$$
\chi=\frac{P}{I U}=\frac{I R}{U}=\frac{I R \sqrt{2}}{U_{1 m}} .
$$

По приведенным формулам были определены выходные параметры схемы $I, I_{\mathrm{cp}}, K_{\phi}$ и $\chi$ в зависимости от угла регулирования $\omega t_{s}$ и величины индуктивности $L$. Для расчетов была использована ЭВМ «Наири К». Неизменными величинами были приняты $U_{1 m}=100 \mathrm{~B}, R=$ $=10$ Ом, $\omega=3141 /$ сек.

Результаты расчетов показаны на рис. 3 , где $I^{*}=I / I_{\text {макс }}$ - относительный уровень выходного тока. В заштрихованной области (ограниченной пунктирными линиями) нечетные гармоники тока не подавляются дросселем - существует эффект сохранения тока в короткозамкнутом контуре. В этой области возможны первые три режима работы тиристорного регулятора. 


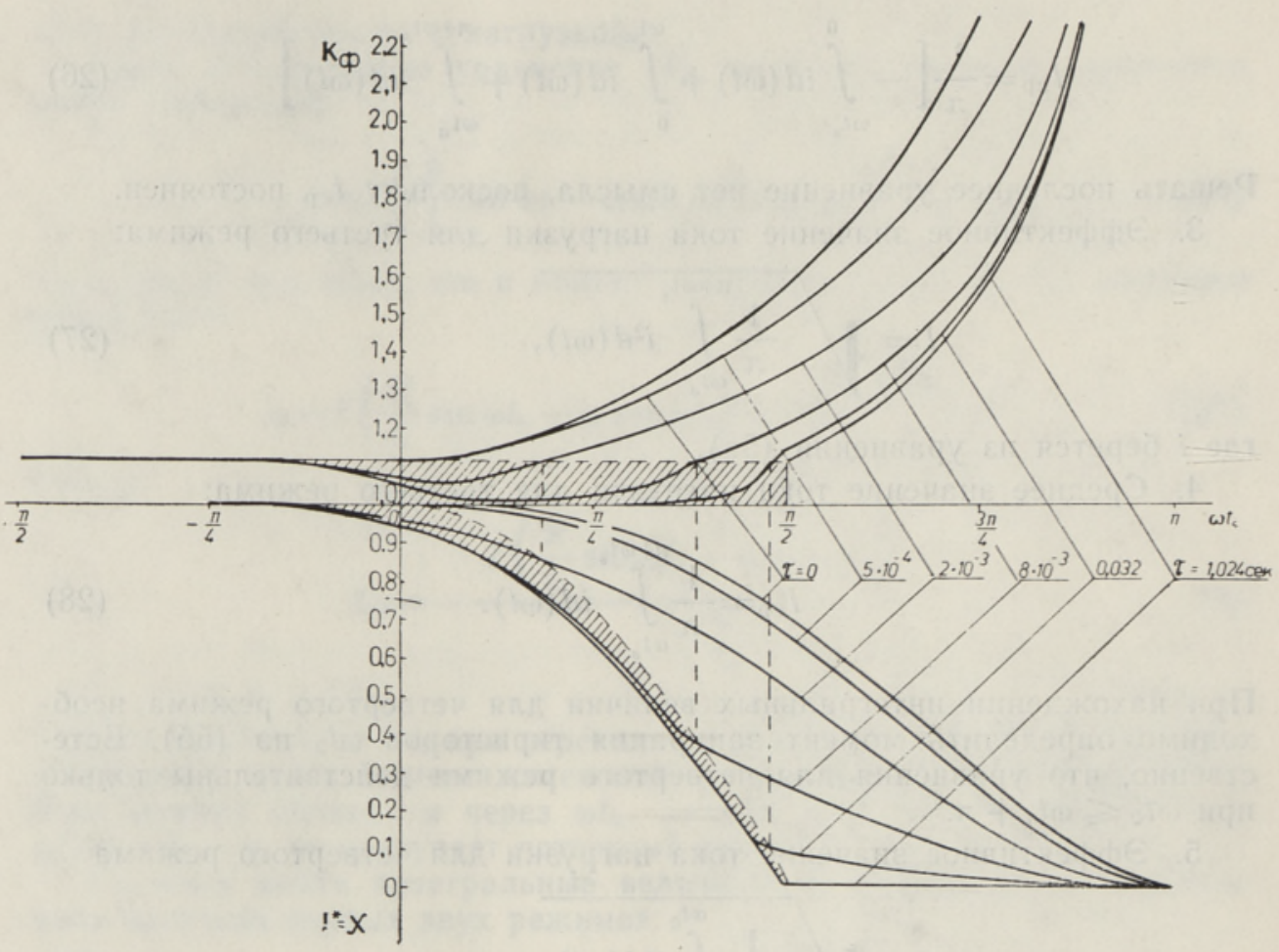

Рис. 3.

\section{Выводы}

1. Рассмотренная схема тиристорного регулятора переменного напряжения обеспечивает существенное расширение диапазона регулирования при углах отпирания тиристоров $0<\omega t_{s}<\pi / 2$, что значительно упрощает управление тиристорами.

2. Коэффициент формы выходного напряжения может изменяться только в пределах

$$
1,0 \leqslant K_{\Phi} \leqslant 1,11 .
$$

3. Постоянная времени тиристорного регулятора при расчетах выбирается в зависимости от желаемого диапазона регулирования.

\section{Л ИТЕ РА Т У Р А}

1. Erlicki, M. S., Em a nuel-Eigeles, A., IEEE Trans. on Industry and General Applications, IGA-4, No. 4, 447 (1968).

2. Le p p, V. R., S ibg at u lin, K. M., C h e rk a s ov, J. N., Current stabilizer having a saturable reactor in the mode of forced magnetization, United States Patent No. 3845380 , Oct. 29,1974 .

3. О я в э р М., С ар в В., Изв. АН ЭССР, Физ. Матем., 25, 392 (1976).

Институт термофизики и электрофизики Академии наук Эстонской ССР

\section{Поступила в редакцию} $14 / \mathrm{IV} 1976$ 
H. TEHVER

\section{LINEAARSE DROSSELI INDUKTIIVSUSE MOJU TOKESTATUD \\ PAARISHARMOONILISTEGA VAHELDUVPINGE-TURISTORREGULAATORI OMADUSTELE}

Analüüsitakse lineaarse drosseli induktiivsuse mõju koormusvoolu kujule. On leitud suhtelise koormusvoolu, võimsusteguri ja kujuteguri sõltuvus reguleerimisnurgast mitmesuguste lineaarse drosseli suhtelise induktiivsuse väärtuste korral.

\section{H. TEHVER}

INFLUENCE OF LINEAR COIL INDUCTANCE ON PROPERTIES OF THE A-C VOLTAGE THYRISTOR REGULATOR WITH SUPPRESSED EVEN HARMONICS

The influence of linear coil inductance on the wave-form of load current is analysed. Expressions of the relative load current, power and the form factor in dependence on the control angle for various values of relative inductance of linear coil, are presented. 